\title{
ESTADO NUTRICIONAL Y ACTIVIDAD FÍSICA EN ESTUDIANTES DE LA ESCUELA DE OBSTETRICIA DE LA UNIVERSIDAD DE GUAYAQUIL, AÑO 2011
}

\author{
NUTRITIONAL STATEAND PHYSICAL ACTIVITY OF STUDENTS FROM THE SCHOOL OF OBSTETRICS \\ OF THE UNIVERSITY OF GUAYAQUIL, YEAR 2011
}

\author{
ESTADO NUTRICIONALE ATIVIDADE FÍSICA EM ESTUDANTES DA ESCOLA DE OBSTETRÍCIA DA UNIVERSIDADE \\ DE GUAYAQUIL, ANO 2011
}

\author{
SÓCRATES POZO VERDESOTO', DELIA CRESPO ANTEPARA', JULLÁN VARAS GAVILANES', OMAR VARGAS SALAS² \\ ${ }^{1}$ Universidad de Guayaquil. Guayaquil, Ecuador \\ ${ }^{2}$ Centro de Salud Martha de Roldós del Ministerio de Salud Pública, Guayaquil, Ecuador.
}

Resumen

Introducción: los estudiantes universitarios constituyen una población vulnerable al desarrollo de alteraciones en su estado nutricional, por características como permanecer muchas horas de clases en actitud sedentaria, y consumir alimentos de preparación rápida y obesogénica, de bares 0 comedores cercanos. Objetivo: determinar el estado nutricional y la práctica de actividad física en estudiantes de la Escuela de Obstetricia de la Universidad de Guayaquil. Materiales y métodos: estudio de diseño no experimental, descriptivo y transversal. Participaron 670 alumnos de la Escuela de Obstetricia de la Facultad de Ciencias Médicas de la Universidad de Guayaquil durante el año 2016, Se realizó evaluación de Índice de Masa Corporal, circunferencia abdominal, y encuesta sobre práctica de actividad física. Resultados: el $44 \%$ de los estudiantes presentó malnutrición, distribuida de la siguiente manera: el $9 \%$ con bajo peso, el $25 \%$ con sobrepeso, y el $10 \%$ con obesidad. El $35 \%$ de las mujeres presentaron valores de circunferencia abdominal relacionadas con riesgo de enfermedad cardiovascular frente al $23 \%$ de los hombres. El $71 \%$ de los estudiantes refirió no realizar actividad física. Discusión: los estudiantes universitarios tuvieron una prevalencia de sobrepeso y obesidad mayor que los reportados por otros estudios similares realizados en la región. El riesgo cardiovascular según circunferencia abdominal, fue mayor para el sexo femenino. La mayoría de la población estudiantil es sedentaria, con valores muy superiores al de otros estudios análogos. Conclusiones: la población de estudiantes presenta problemas de malnutrición principalmente por exceso, a pesar de tener conocimientos sobre nutrición. Es necesario identificar factores determinantes que están influyendo en ellos, para fortalecer programas que mejoren los hábitos nutricionales y de actividad física.

PALABRAS CLAVE: estado nutricional, circunferencia abdominal, actividad física.

Abstract

Introduction: university students are a vulnerable population in the development of complications in their nutritional status for reasons such as being sedentary for long class hours and eating fast and obesogenic food sold at cafeterias or university canteens. Oobjective: to determine the nutritional status and the practice of physical activities among students from the School of Obstetrics at the University of Guayaquil. Materials and methods: non experimental, descriptive and transversal study. Sample population was made up by 670 students from the School of Obstetrics of the Medical Faculty at the University of Guayaquil during the year 2016. Assessment of Body Mass Index, abdominal girth, and survey about practice of physical activity were carried out. Results: $44 \%$ of the students presented malnutrition, distributed in the following way: $9 \%$ low weight, $25 \%$ overweight, and $10 \%$ obesity. $35 \%$ of women presented waist circumference related with risks of cardiovascular diseases compared to $23 \%$ of men. $71 \%$ of the students admitted not doing physical activity. Discussion: University students had a prevalence of overweight and obesity higher than others reported in similar studies made in the region. Cardiovascular risk according to waist circumference was higher among females. The majority of the students is sedentary, with higher numbers compared to similar studies. Conclusions: this population of students presents malnutrition problems mainly due to excess, despite having knowledge about nutrition. It is necessary to identify factors that persuade them to have this behavior, and strengthen programs that help improve nutritional habits and physical activity.

KEYWORDS: nutritional status, abdominal circumference, exercise.

Resumo

INTRODUÇÃo: os estudantes universitários constituem uma população vulnerável ao desenvolvimento de alterações no seu estado nutricional, pelas características como permanecer por muitas horas em aulas, em atitude sedentária e pelo consumo de alimentos de preparação rápida e obesogenica, de bares e lanchonetes próximos. Objetivo: determinar 0 estado nutricional e a pratica de atividades físicas em estudantes da Escola de Obstétrica da Universidade de Guayaquil. Materiais e métodos: estudo de desenho não experimental, descritivo e transversal. 670 alunos da Escola de Obstetrícia da Facultade de Ciências Medicas formaram parte do estudo no ano de 2016. Uma avaliação do índice de massa corporal foi feita, assim como medição de circunferência abdominal e um questionário sobre a pratica de atividade física. Resultados: Cerca de $44 \%$ dos estudantes apresentaram malnutrição, distribuída da seguinte maneira: Cerca de $9 \%$ falta de peso, $25 \%$ sobrepeso, e $10 \%$ obesidade. Cerca de $35 \%$ das mulheres apresentaram valores de circunferência abdominal relacionadas com risco de enfermidade cardiovascular frente a $23 \%$ dos homens. Cerca de $71 \%$ dos estudantes indicaram não realizar atividade física alguma. Discussão: os estudantes universitários tiveram uma prevalência de sobrepeso e obesidade maior que os reportados por outros estudos semelhantes na região. 0 risco cardiovascular, segundo a circunferência abdominal, foi maior para o sexo feminino. A maioria da população estudantil apresenta problemas de malnutrição principalmente por excesso, a pesar de ter conhecimentos de nutrição. É necessário identificar os fatores determinantes que os estão influenciando, para fortalecer programas que melhorem os hábitos nutricionais e de atividade física

PALABRAS-CHAVE: estado nutricional, circunferência abdominal, exercício. 
INTRODUCCIÓN

Según la Encuesta Nacional de Salud y Nutrición ENSANUT-ECU 2012, la prevalencia de adultos con delgadez es $1.3 \%$, y la prevalencia de sobrepeso y obesidad en Ecuador es $62.8 \%$. La obesidad es más alta en el sexo femenino (27.6 \%) que en el masculino (16.6\%), al contrario del sobrepeso, donde el sexo masculino tiene una prevalencia de $43.4 \%$ y el femenino de $37.9 \%$. Sin embargo la población considerada en ese estudio, estuvo en las edades comprendidas entre 19 y 60 años. ${ }^{1}$ Por otro lado en relación a la actividad física, en mismo estudio establece como conclusión general que en todos los grupos poblacionales los niveles de inactividad y sedentarismo son bastante altos, mientras la proporción de ecuatorianos que llevan una vida activa es inaceptablemente baja. ${ }^{1}$

El estudiante universitario, es muy vulnerable al desarrollo de alteraciones en su estado nutricional, por las características propias, como la de permanecer mayor número de horas sentado, recibiendo clases, o de tener un horario de clases que ocupa casi todo su día, sumando a esto las horas que deberá dedicarlas al estudio en su casa. Factores que llevan a que se incremente el sedentarismo. También la mayoría deberá alimentarseen los bares o comedores de la universidad o cercanos a ella, por cuestión de horarios o porque simplemente vienen de otros lugares del país y viven solos, sin tiempo para preparar sus alimentos. ${ }^{2}$

Con estos antecedentes se realiza un estudio para conocer el estado nutricional de estudiantes de la Escuela de Obstetricia de la Facultad de Ciencias Médicas de la Universidad de Guayaquil, estableciendo el índice de masa corporal y la clasificación sugerida por la Organización Mundial de la Salud; también determinar el grado de actividad física mediante la aplicación de encuestas.

\section{MATERIALES Y MÉTODOS}

Se realizó un estudio de diseño observacional, de tipo descriptivo y transversal, en la Escuela de Obstetricia, de la Facultad de Ciencias Médicas de la Universidad de Guayaquil, durante los meses de enero a marzo del año 2016, para determinar el estado nutricional y la práctica de actividad física de 670 estudiantes, que participaron voluntariamente, excluyéndose: los que faltaron el día de la evaluación, los que no desearon participar y las alumnas embarazadas.

El estudio consideró las recomendaciones de la Declaración de Helsinski. Se realizó evaluación antropométrica que incluyó peso, talla, Índice de
Masa Corporal según la fórmula de Quetelet (peso/ talla 2), circunferencia abdominal, y encuesta sobre práctica de actividad física, que determinó el tiempo diario y días de la semana destinados a este fin. La talla se obtuvo midiendo con cinta métrica adosada a la pared, con el nivel 0 en el piso, el estudiante con los pies descalzos y unidos, se colocaba de espaldas a la pared, tocando esta en cuatro puntos: talones, glúteos, escápulas, y cabeza, la que seguía el plano deFrankfort. Elpeso semidió en una báscula marca SECA con una precisión de $0,1 \mathrm{~kg}$. La circunferencia abdominal se midió con cinta métrica inextensible, considerando el punto equidistante entre la espina iliaca y la última costilla como referencias, se realizaban tres medidas, de las que se obtenía su promedio, el queseconsideraba comovalordeestudio, y clasificado según lo dispuesto por la International Diabetes Federation (IDF), como de riego para el desarrollo de enfermedades cardiovasculares si el valor era mayor de $80 \mathrm{~cm}$ para el sexo femenino, y de $94 \mathrm{~cm}$ para el sexo masculino. Los datos fueron tabulados y expresados en frecuencias absolutas, porcentajes para las variables cualitativas. En las variablescuantitativas secalculóla media, desviación estándar (DE) y rango. Se empleó la prueba Chi cuadarado, con un nivel de significancia $\leq 0.05$, para determinar asociación entre las variables. Los resultados fueron expuestos en tablas y gráficos usando el programa Excel de Microsoft Office de Windows $10^{\circledR}$.

\section{RESULTADOS}

El estudio contó con la participación de 670 estudiantes, de los cuales 576 eran mujeres y 94 hombres. La media de edad fue 21.8 años con una DE de 3.2, la edad mínima fue de 17 años y la máxima de 42 años. Se encontró que el 56\% (376) de la población de estudiantes tenía un Índice de Masa Corporal considerado normal, el 44\% (294) restante tenía problemas de malnutrición, distribuidos de la siguiente manera: el 9\% (61) con bajo peso, el 25\% (168) con sobrepeso, y el 10\% (65) con obesidad (figura 1).

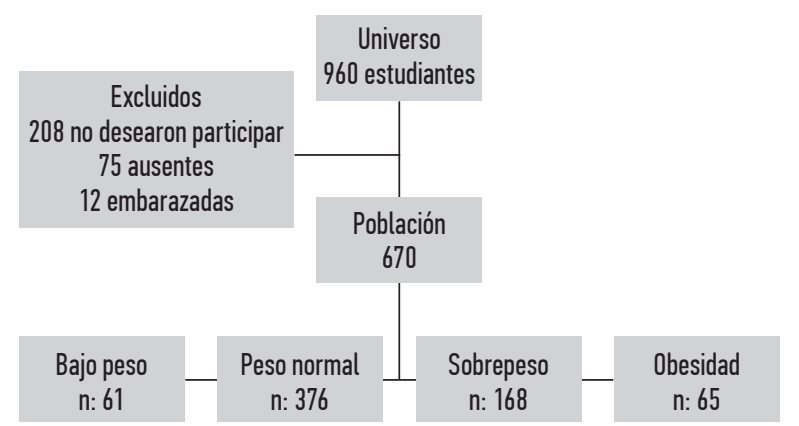

Figura 1. Flujograma del estado nutricional de los estudiantes de la Escuela de Obstetricia de la Universidad de Guayaquil. 
En relación a la distribución del estado nutricional de acuerdo al sexo se encontró peso normal en el $57 \%$ (330) de las mujeres y en el $48 \%$ (46) de los hombres ( $\mathrm{p}=0.105)$; con bajo peso el $10 \%$ (56) de las mujeres y el $5 \%$ (5) de los hombres (p $=0.178$ ); sobrepeso $37 \%$ (34) de los hombres y el $23 \%$ (134) de las mujeres ( $\mathrm{p}=0.006)$; obesidad el $10 \%$ para ambos sexos (mujeres 56 y hombres 9 ) $(\mathrm{p}=0.993)$. (figura 2).

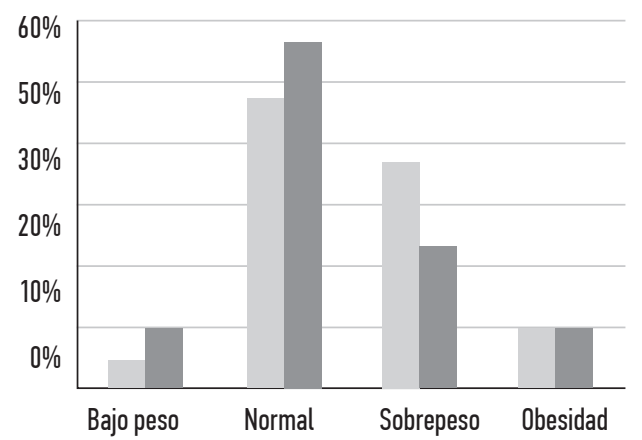

Figura 2. Distribución del estado nutricional según sexo.

Con respecto a la distribución de estado nutricional según año académico, no se observaron diferencias significativas $(\mathrm{p}=0.623)$ (tabla 1$)$.

TABLA 1. DISTRIBUCIÓN DE ESTADO NUTRICIONAL POR AÑO ACADÉMICO

\begin{tabular}{|c|c|c|c|c|c|c|c|c|}
\hline \multirow[t]{2}{*}{$\begin{array}{l}\text { AÑ̃ } \\
\text { ACADÉMICO }\end{array}$} & \multicolumn{2}{|c|}{ BAJO PESO } & \multicolumn{2}{|c|}{$\begin{array}{r}\text { PESO } \\
\text { NORMAL }\end{array}$} & \multicolumn{2}{|c|}{ SOBREPESO } & \multicolumn{2}{|c|}{ OBESIDAD } \\
\hline & 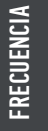 & 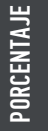 & 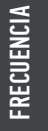 & 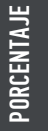 & 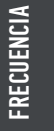 & 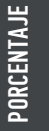 & 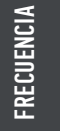 & $\begin{array}{l}\text { 崖 } \\
\text { 息 } \\
\text { 总 }\end{array}$ \\
\hline 1 año & 26 & 10 & 149 & 55 & 64 & 24 & 29 & 11 \\
\hline 2 año & 15 & 10 & 87 & 60 & 32 & 22 & 12 & 8 \\
\hline 3 año & 16 & 10 & 88 & 56 & 38 & 24 & 15 & 10 \\
\hline 4 año & 3 & 8 & 21 & 52 & 13 & 31 & 3 & 9 \\
\hline 5 año & 1 & 2 & 31 & 51 & 21 & 35 & 6 & 12 \\
\hline
\end{tabular}

Según los valores de circunferencia abdominal relacionados con riesgo de enfermedad cardiovascular definidos por la International Diabetes Federation (IDF): el 35\% (201) de las mujeres tienen valores de riesgo; mientras el 23\% (22) de los hombres tuvo esta condición; diferencia que resultó significativa $(\mathrm{p}=0.028)$ (tabla 2$)$.

TABLA 2. DISTRIBUCIÓN DE RIESGO SEGÚN CIRCUNFERENCIA ABDOMINAL EN HOMBRES Y MUJERES

\begin{tabular}{lrrrr} 
& \multicolumn{2}{c}{ MUJERES } & \multicolumn{2}{c}{ HOMBRES } \\
& FRECUENCIA & PORCENTAJE & FRECUENCIA & FRECUENCIA \\
Sin riesgo & 375 & $65 \%$ & 72 & $77 \%$ \\
Con riesgo & 201 & $35 \%$ & 22 & $23 \%$ \\
\hline Total & 576 & $100 \%$ & 94 & $100 \%$ \\
\hline
\end{tabular}

En cuanto a la actividad física, el $71 \%$ (476) de los estudiantes refirió no realizarla, y apenas un $29 \%$ (194) practicaba algún tipo de actividad física, con una media de 3.27 días a la semana y una desviación estándar de 2,1. La distribución del tiempo diario destinado a la actividad física fue de $72 \%$ entre 30 minutos a una hora, $15 \%$ más de una hora, y $13 \%$ menos de 30 minutos.

\section{DISCUSIÓN}

De los estudiantes evaluados en el presente estudio el $56 \%$ tenía un índice de masa corporal considerado adecuado, cifra menor al encontrado en estudiantes universitarios de la Región de Murcia (España), que fue de $75 \%$ y 80,2\%.3,4 En dos estudios realizados en estudiantes universitarios de Bogotá (Colombia), también se reportaron porcentajes de peso normal, mayores que los del presente estudio. 5,6 Otros estudios realizados con estudiantes de Universidades Mexicanas presentan resultados con mayor similitud a los de este trabajo. ${ }^{7-9}$ Los estudiantes de la Escuela de Obstetricia de la Universidad de Guayaquil tienen una prevalencia de sobrepeso y obesidad del $35 \%$, y de bajo peso del $9 \%$, valores con tendencia a incrementarse si consideramos los reportados por la Encuesta Nacional de Salud y Nutrición ENSANUT-ECU 2012 para la población adulta ecuatoriana que es del $62.8 \%$ y del $1,3 \%$ respectivamente. ${ }^{1}$

En relación con el sexo, en este estudio se obtuvieron resultados similares a los reportados en varios estudios, siendo el sobrepeso más común en los hombres y el bajo peso más frecuente en las mujeres. ${ }^{10-13}$.

Se encontró que el $33 \%$ de todos los estudiantes tenían valores de riesgo cardiovascular por incremento en su perímetro abdominal. La distribución de casos según sexo estaba en 35\% de las mujeres y el $23 \%$ de los hombres. Estos valores fueron superiores a los hallados en otros estudios. ${ }^{4,8}$

El $71 \%$ de los estudiantes encuestados en este estudio refirieron no realizar actividad física, valor muy superior al reportado en estudiantes universitarios de la región. 3,14,15 Esto es importante porque la inactividad física, según la Organización Mundial de la Salud, se encuentra entre los 10 principales factores de riesgo de mortalidad a nivel mundial. ${ }^{17}$ 
Entendemos que la educación y el conocimiento no son la principal barrera para tener un peso sano. Al tratarse de estudiantes de una carrera universitaria relacionada con el Área de la Salud, que poseen conocimientos sobre un estilo de vida saludable, que incluye una nutrición balanceada y actividad física; es que asumimos que otros factores podrían ser los determinantes, y no el conocimiento, esta problemática del peso en los universitarios. Conocer esto es importante para el momento que se desee implementar programas de mejoramiento de la salud dentro de las universidades.

\section{CONCLUSIONES}

Los estudiantes universitarios de la Escuela de Obstetricia de la Universidad de Guayaquil presentan problemas de malnutrición, sobre todo por exceso; existe además riesgo de enfermedad cardiovascular a predominio significativo del sexo femenino. La mayoría de estudiantes no realizan actividad física. Al ser estudiantes del Área de la Salud, con conocimientos de nutrición, habrá que considerar otros determinantes que intervienen en esta problemática, para fortalecer programas que mejoren las salud estudiantil.

\section{REFERENCIAS BIBLIOGRÁFICAS}

1. Freire WB, Ramírez-Luzuriaga MJ, Belmont P, Mendieta MJ, Silva-Jaramillo MK, Romero N, et al Tomo I: Encuesta Nacional de Salud y Nutrición de la población ecuatoriana de cero a 59 años. ENSANUT-ECU 2012. Ministerio de Salud Pública/Instituto Nacional de Estadísticas y Censos. 1ª ed Quito-Ecuador; 2014. p. 248-254

2. Montero A, Úbeda N, García A. Evaluación de los hábitos alimentarios de una población de estudiantes universitarios en relación con sus conocimientos nutricionales. Nutr Hosp. 2006;21(4):466-73

3. Cutillas A, Herrero E, Eustaquio A, Zamora S, y Pérez-Llamas F. Prevalencia de peso insuficiente, sobrepeso y obesidad, ingesta de energía y perfil calórico de la dieta de estudiantes universitarios de la Comunidad Autónoma de la Región de Murcia (España) Nutr Hosp. 2013;28(3):683-689.

4. Rizo M, González N, Cortés E. Calidad de la dieta y estilos de vida en estudiantes de Ciencias de la Salud. Nutr Hosp. 2014;29(1):153-157

5. Vargas M, Becerra F, Prieto E. Evaluación Antropométrica de Estudiantes Universitarios en Bogotá, Colombia. Revista de Salud Pública. 2008;10 (3):433-442

6. Becerra F, Pinzón G, Vargas M. Estado nutricional y consumo de alimentos de estudiantes universitarios admitidos a la carrera de medicina, Bogotá 20102011. Rev Fac Med. 2012;60(1): 762-75
7. Salazar C, Feu S, Vizuete M, De la Cruz E. IMC y actividad física de los estudiantes de la Universidad de Colima. Rev. Inter. de Medicina y Ciencias de la Actividad Física y el Deporte 2013;13(51):569-584

8. Mollinedo Montaño Fabiana Esther, Trejo Ortiz Perla María, Araujo Espino Roxana, Lugo Balderas Lilia Guadalupe. Índice de masa corporal, frecuencia y motivos de estudiantes universitarios para realizar actividad física. Educ Med Super 2013;27(3):189-199

9. Rodríguez L, Rodríguez. Percepción de la imagen corporal, índice de masa corporal y sobrepeso en estudiantes universitarios del Sureste. Rev. mex. de Pediatría. 2001;68(4):135-138

10. Lorenzini R, Betancour D, Chel L, Segura M, Castellanos A. Estado Nutricional en relación a estilos de vida de estudiantes universitarios mexicanos. 2015;32 (1): 94-100

11. Mardones M, Olivares S, Araneda J, Gómez N. Etapas del cambio relacionadas con el consumo de frutas y verduras, actividad física y control del peso en estudiantes universitarios chilenos. Archivos latinoamericanos de nutricion. 2009;59(3):304.

12. Martínez C, Veiga P, López A, J. Cobo M, Carbajal A. Evaluación del estado nutricional de un grupo de estudiantes universitarios mediante parámetros dietéticos y de composición corporal. Nutr. Hosp. (2005);20(3):197-203

13. Magallanes M, Gallegos E, Carrillo A, Sifuentes D, Olvera M. Sobrepeso, obesidad y dislipidemias en población universitaria del noreste de México. Rev. Inv. y Educación en Enfermería. 2010;28(19):100-107

14. Ledo M, Román D, González M, Izaola O, Conde R, De la Fuente R. Características nutricionales y estilo de vida en universitarios. Nutr Hosp. 2011;26(4):814818

15. Herazo Y, Hernández J, Domínguez R. Etapas de cambio y niveles de actividad física en estudiantes universitarios de Cartagena (Colombia). Salud Uninorte. 2012;28(2):298-318.

16. Varela M, Duarte C, Salazar I, Lema L, Tamyo J. Actividad física y sedentarismo en jóvenes universitarios de Colombia: prácticas, motivos, y recursos para realizarlas. Colombia Médica. 2011;42:269-77

17. Organización Mundial de la Salud. Centro de Prensa: Nota descriptiva $\mathrm{N}^{\circ} 384$ Junio de 2016. Disponible en http://www.who.int/mediacentre/factsheets/ fs385/es/ 